\title{
Metastatic endophthalmitis and thyroid abscess complicating Klebsiella pneumoniae liver abscess
}

\author{
Seon-Jae Kim, Seong Taek Chu, Keun Sook Lee, Seung Woo Nam, Jong Kyoung Choi, Joo Won Chung, \\ and Hyeok Choon Kwon
}

Department of Gastroenterology and Hepatology, National Medical Center, Seoul, Korea

The thyroid is resistant to infection due to its anatomical and physiological characteristics. We present a rare case of invasive liver abscess with metastatic thyroid abscess and endogenous endophthalmitis in a previously healthy 55 -yearold female patient without diabetes or other medical illness. This report raises an important question of the virulence of Klebsiella pneumoniae as an increasingly common causative agent of liver abscess. (Clin Mol Hepatol 2018;24:88-91) Keywords: Endophthalmitis; Thyroid abscess; Klebsiella pneumoniae; Liver abscess

\section{INTRODUCTION}

The prevalence of Klebsiella pneumoniae (K. pneumoniae) infection has been increasing since the late 1980s when anecdotal reports were published. ${ }^{1-3}$ Now $K$. pneumoniae is the predominant pathogen in more than half of all liver abscesses. ${ }^{4}$ Fang et al. ${ }^{5}$ define invasive liver abscess syndrome as K. pneumoniae abscess with extrahepatic complications, resulting in severe complications and poor outcomes. ${ }^{2,6}$ Extrahepatic complications resulting from bacteremic dissemination are reported frequently, including endophthalmitis, meningitis and necrotizing fasciitis. ${ }^{7,8}$ We present a rare case of invasive liver abscess syndrome due to K.pneumoniae with metastatic endophthalmitis and thyroid abscess. The patient was a healthy 55-year-old female without any prior illness or predisposing hepatobilliary disease. Although the thyroid is resistant to infection, she presented with two distant metastatic infections. This report raises the importance of consideration of metastatic infection during treatment of liver abscess.

\section{CASE REPORT}

A 55-year-old female presented with left flank pain for one day. She was healthy and denied any illness. She was not being prescribed any medicine and rarely consumed alcohol. She denied travel history and taking any herbal medicine.

Her vital signs were unremarkable. Laboratory tests revealed the following results: aspartate aminotransferase (AST) level, 66 $\mathrm{U} / \mathrm{L}$ (normal range, 0-40 U/L); alanine aminotransferase (ALT) level, $74 \mathrm{U} / \mathrm{L}$ (normal range, 0-45 U/L); alkaline phosphatase (ALP) level, $186 \mathrm{U} / \mathrm{L}$ (normal range, 30-130 U/L); total bilirubin level, 1.4 $\mathrm{U} / \mathrm{L}$ (normal range, 0.2-1.2 U/L); WBC level $14,600 / \mathrm{mm}^{3}$ and c-reactive protein (CRP) level $275 \mathrm{U} / \mathrm{L}$ (normal range, 0-3.0 U/L). Serologic tests for hepatitis $B$ and $C$ viruses and HIV antigen test were negative. Thyroid function test was normal.

Abdominal CT scans demonstrated a $14.0 \times 6.5 \mathrm{~cm}$ sized lobulated, hypoechoic lesion in segment 2 and showed resolution after percutaneous transhepatic drainage (Fig. 1). On the second day

\section{Abbreviations:}

ALT, alanine aminotransferase; ALP, alkaline phosphatase; AST, aspartate aminotransferase; CT, computed tomography; CRP, c-reactive protein; K. pneumoniae, Klebsiella pneumoniae; WBC, white blood cell

\section{Corresponding author: Hyeok Choon Kwon}

Department of gastroenterology and hepatology, National Medical Center, 245 Eulji-ro, Jung-gu, Seoul 04564, Korea

Tel: +82-2-2260-7431, Fax: +82-2-2267-8685

E-mail:hchkwon@naver.com

http://orcid.org/0000-0003-3740-3033 

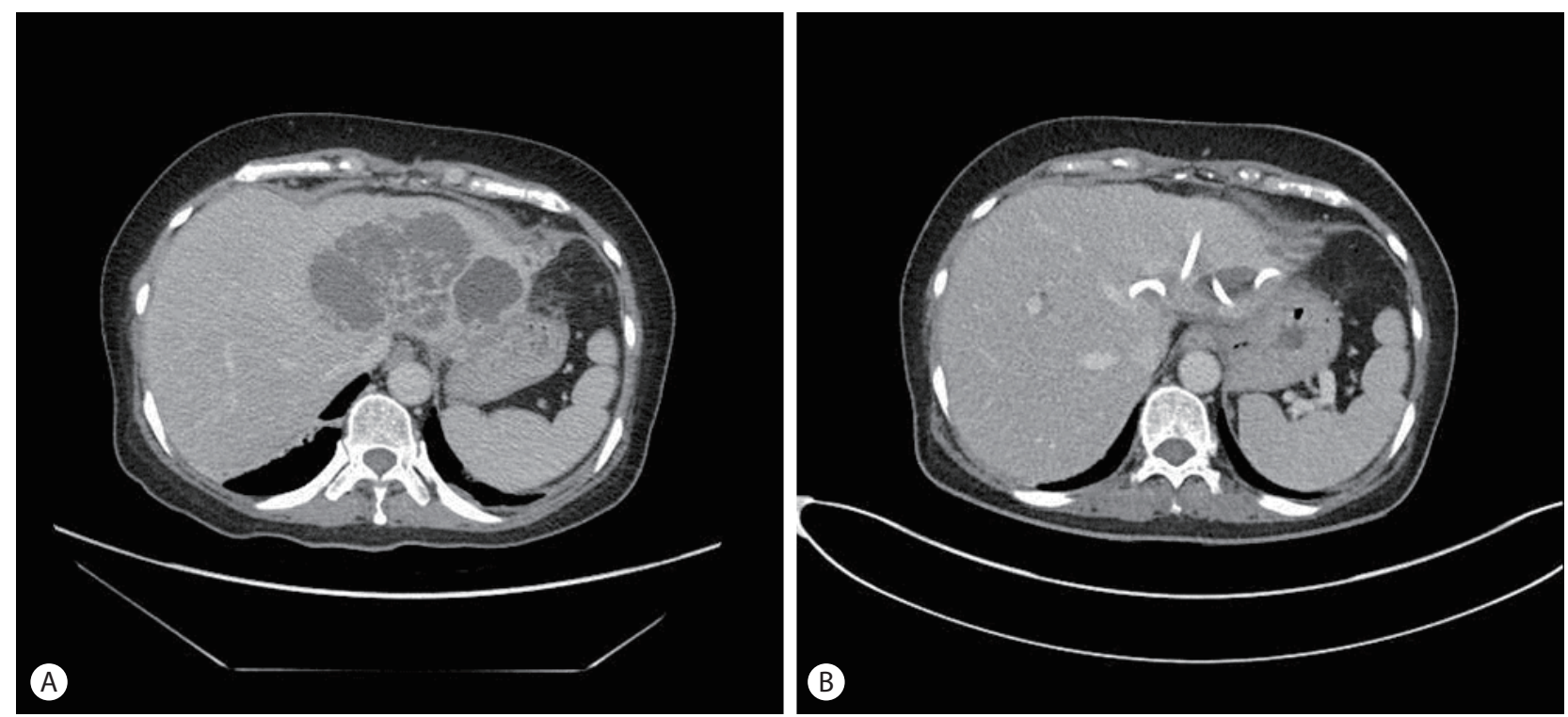

Figure 1. Computed tomographic (CT) scan. (A) Abdominal CT scans demonstrated a $14 \times 6.5 \mathrm{~cm}$ sized Hypoechoic lesion in segment 2. (B) CT scans showed resolution after percutaneous transhepatic drainage.
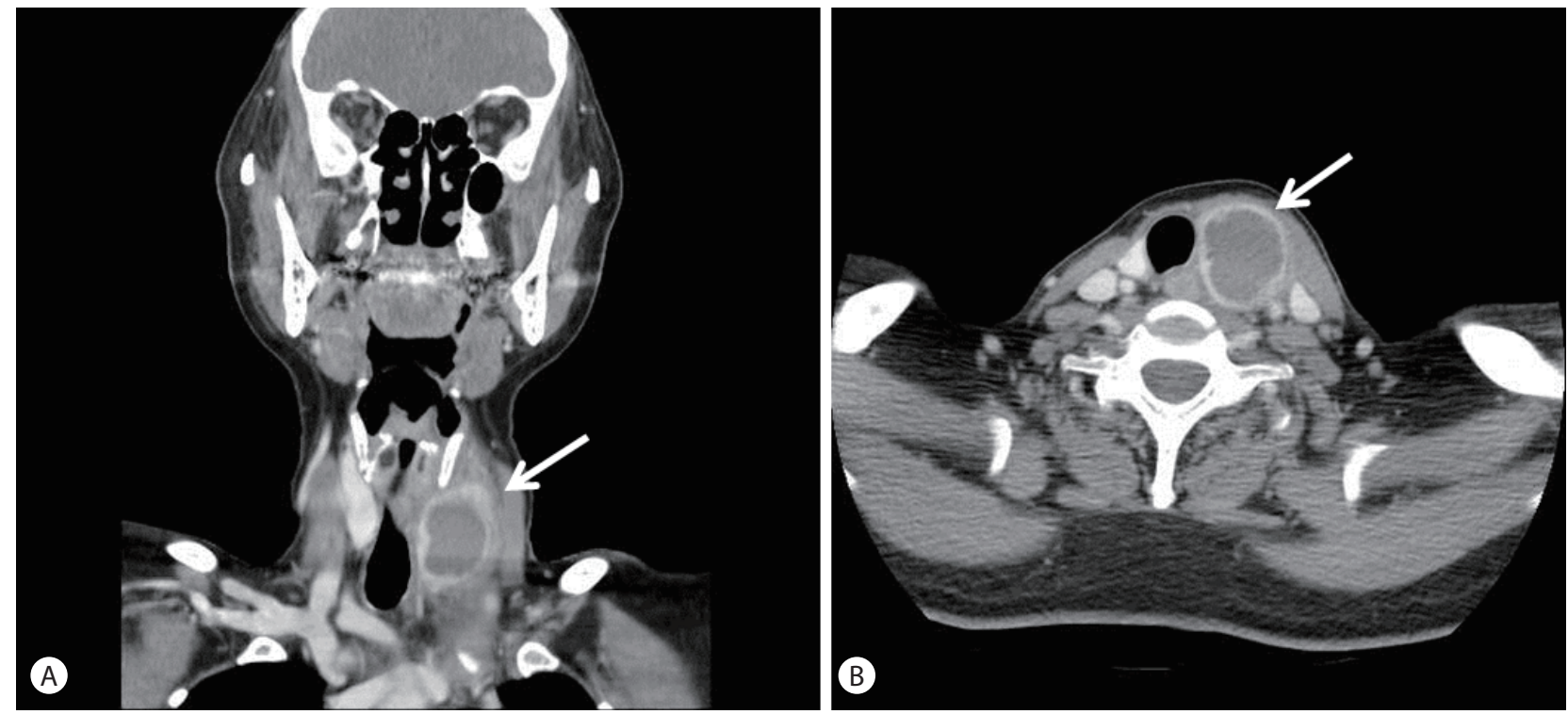

Figure 2. CT scan of the neck showed a $3.5 \times 2.5 \mathrm{~cm}$ sized structure of low density, with heterogenous contents, including fluid, with dense wall and light contrast enhancement (arrow), (A) coronal, (B) axial.

after admission, the patient complained of visual disturbance and ophthalmic pain. She was diagnosed with metastatic endophthalmitis, and received intravitreal vancomycin and ceftazidime injection. On hospitalization day (HOD) 3 and HOD 8, she was treated with vitrectomy and lensectomy. On HOD 9, she complained of painful swelling with erythema on the left anterior aspect of her neck. CT scan of the neck showed a $3.5 \times 2.5 \mathrm{~cm}$ sized heterogenous low density structure filled with fluid and wall enhancement by contrast (Fig. 2). Thyroid ultra-sound (US) revealed $3.5 \times 2.5 \mathrm{~cm}$ sized well-defined huge abscess which was resolved after drainage (Fig. 3). Ultra-sound (US) guided aspiration was performed for diagnosis and drainage was done twice on HOD 10 and HOD 13. She started antibiotic treatment with cefotaxime, metronidazole and amikacin at admission. K. pneumoniae was cultured from patient's blood, liver aspirate and thyroid aspirate. The antibiotics was changed to cefotaxime only after isolating $K$. pneu- 

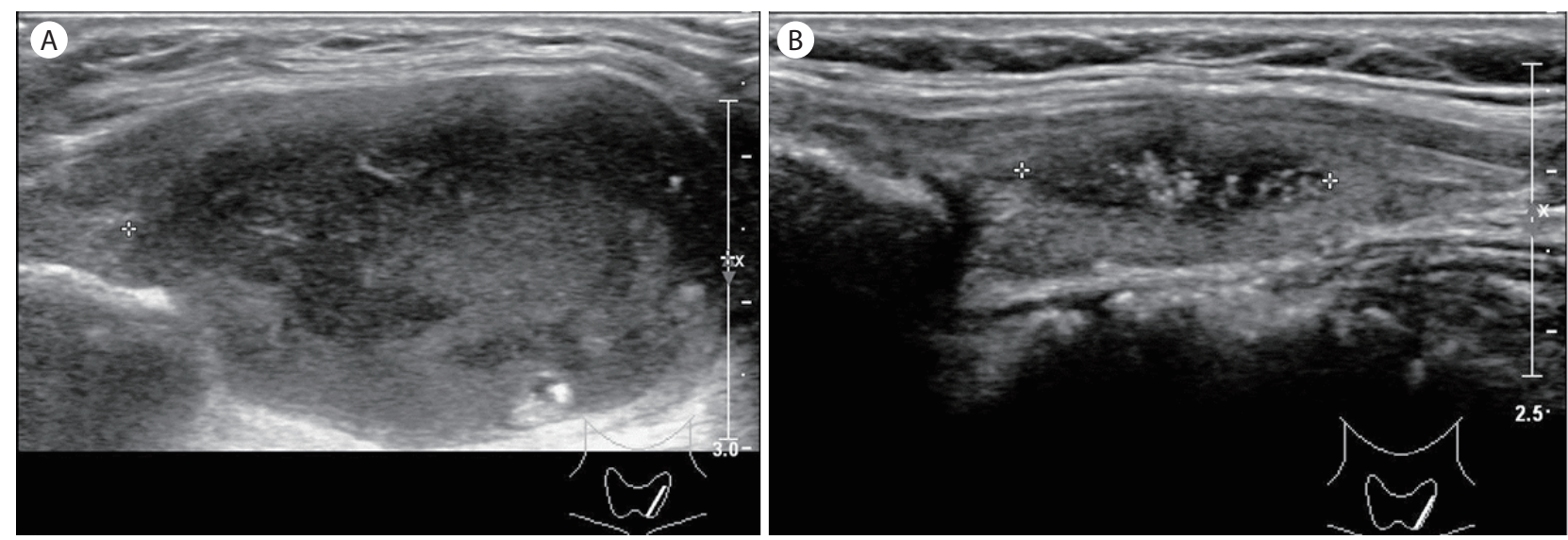

Figure 3. Thyroid Ultra-sound (US). (A) Ultra-sound (US) demonstrated a $35 \times 25 \mathrm{~mm}$ sized well-defined huge abscess. (B) Ultra-sound (US) showed resolution after drainage.

moniae as the causative pathogen on culture. She was discharged on HOD 37 after a CT demonstrating resolution of liver and thyroid abscess and improving fever and CRP. However, she lost her right visual acuity on discharge.

\section{DISCUSSSION}

Klebsiella pneumoniae is a gram-negative, non-motile, lactose fermenting, rod-shape organism.

This organism can be found in the mouth, skin, and intestinal tract, where it initially does not cause disease. But, it can progress into severe bacterial infections leading to pneumonia, urinary tract infections or meningitis. Haematogenous seeding has been considered as a possible route of septic metastases from pyogenic liver abscess. ${ }^{9}$

In the past decades, prevalence of invasive liver abscess syndrome, KPLA with extrahepatic complications has increased in Asia. Taiwan has the highest prevalence, followed by South Korea. ${ }^{5,8}$ The invasive nature of some $K$. pneumoniae strains includes a hypermucoviscous phenotype associated with serotypes $\mathrm{K} 1$ and $\mathrm{K} 2$ and the regulator of mucoid phenotype $\mathrm{A}$ gene. Almost all patients with severe infection with bacteremia, liver abscess, and extrahepatic infections are infected exclusively with $K$. pneumoniae serotypes $\mathrm{K} 1$ and $\mathrm{K} 2$, but not all infections with $\mathrm{K} 1$ or $\mathrm{K} 2$ serotypes result in liver abscess with extrahepatic infection. ${ }^{5}$

Extrahepatic metastases sites include the skin, eyes, kidney, lungs, bones, prostate, muscle, and cerebrospinal fluid. Lungs, CNS, and eyes are the most common metastatic sites. Gram-negative organisms (e.g., E. coli) were other pathogens of liver abscess inducing extrahepatic complication but rare. ${ }^{5,9}$ The meta- static complications in patients with $K$. pneumoniae liver abscess comprising endophthalmitis, central nervous system (CNS) infections and necrotizing fasciitis are usually severe and associated with poor outcomes. ${ }^{7,10,11}$ As the prevalence of liver abscess has increased, the extrahepatic complications including endogenous endophthalmitis have increased in South Korea. ${ }^{12}$ The prognosis for patients with endophthalmitis caused by $K$. pneumoniae is very poor; more than $85 \%$ of patients had a severe visual deficit. $5,7,10,11$

Usually, thyroid abscess is related to a predisposing congenital variant, most commonly a pyriform sinus fistula or complication of a biopsy. ${ }^{13}$ Metastatic thyroid abscess is a rare complication because the thyroid gland is resistant to infection due to its encapsulation, iodine concentration, rich lymphatic drainage and dual blood supply. ${ }^{14}$ Reviews of thyroid abscess document a predisposing congenital variant and immunocompromised status with atypical pathogens. ${ }^{15-17}$ While thyroid abscesses are uncommon, they are associated with significant risk of rapid progression and potential compromise of the airway, so prompt recognition and treatment are essential for improved patient outcomes.

Both host and virulence factors such as diabetes mellitus and $K$. pneumoniae contribute to the pathogenesis of invasive liver $a b$ scess syndrome ${ }^{18}$, but whether diabetes is an independent risk factor is uncertain. This patient was 55-year-old healthy woman without any history of diabetes or immunodeficiency.

In this report, we describe the first case of $K$. peumoniae invasive liver abscess syndrome with thyroid abscess. The patient was a healthy middle-aged woman with no history of diabetes or immunocompromised status. However, she presented with extrahepatic complications at two different sites, endogenous endophthalmitis and thyroid abscess. In spite of prompt proper antibiotics and vitrectomy, she lost her right vision. 
Seon-Jae Kim, et al. Thyroid abscess complicating liver abscess

Yoon reported that $9.9 \%$ of patients with a liver abscess caused by $K$. pneumoniae had an extrahepatic metastatic infection. ${ }^{4}$ Both host and virulence factors contribute, but with this report we advocate that even when the patient has no predisposing risks, the patient can develop severe extrahepatic complications, and we should rapidly detect and urgently treat to avoid devastating complications. Clinical appraisal and action should include detecting bacteria-associated virulent factors, quick capsular serotyping, emergent radiologic evaluation, early adequate drainage and appropriate treatment with antibiotics.

\section{Conflicts of Interest}

The authors have no conflicts to disclose.

\section{REFERENCES}

1. Chang FY, Chou MY, Fan RL, Shaio MF. A clinical study of Klebsiella liver abscess. Taiwan Yi Xue Hui Xa Zhi 1988;87:282-287.

2. Chung DR, Lee SS, Lee HR, Kim HB, Choi HJ, Eom JS, et al. Emerging invasive liver abscess caused by K1 serotype Klebsiella pneumoniae in Korea. J Infect 2007;54:578-583.

3. Kim SB, JE BK, Lee KY, Lee SH, Chung HH, Cha SH. Computed tomographic differences of pyogenic liver abscesses caused by Klebsiella pneumonia and non-Klebsiella pneumonia. J Comput Assist Tomogr 2007;31:59-65.

4. Yoon JH, Kim YJ, Jun YH, Kim SI, Kang JY, Suk KT, et al. Liver abscess due to Klebsiella pneumoniae: risk factors for metastatic infection. Scand J Infect Dis 2014;46:21-26.

5. Siu LK, Yeh KM, Lin JC, Fung CP, Chang FY. Klebsiella pneumonia liver abscess: an new invasive syndrome. Lancet Infect Dis 2012;12:881-887.

6. Fang CT, Lai SY, Yi WC, Hsueh PR, Leiu KL, Chang SC. Klebsiella pneumonia genotype K1: an emerging pathogen that causes septic ocular or central nervous system complications from pyogenic liver abscess. Clin Infect Dis 2007;45:284-293.

7. Hu BS, Lau YJ, Shi ZY, Lin YH. Necrotizing fasciitis associated with Klebsiella pneumonia liver abscess. Clin Infect Dis 1999;29:13601361.

8. Lee SS, Chen YS, Tsai HC, Wann SR, Lin HH, Huang CK, et al. Predictors of septic metastatic infection and mortality among patients with Klebsiella pneumonia liver abscess. Clin Infect Dis 2008;47:642650.

9. Chen SC, Lee YT, Lai KC, Cheng KS, Jeng LB, Wu WY, et al. Risk factors for developing metastatic infection from pyogenic liver $a b$ scesses. Swiss Med Wkly 2006;136:119-126.

10. Chen SC. Huang CC. Tsai SJ, Yen CH, Lin DB, Wang PH, et al. Severity of disease as main predictor for mortality in patients with pyogenic liver abscess. Am J Surg 2009;192:164-172.

11. Tsai FC, Huang YT, Chang LY, Wang JT. Pyogenic liver abscess as endemic disease, Taiwan. Emerg Infect Dis 2008;14:1592-1600.

12. Nam KY, Lee JE, Lee JE, Jeung WJ, Park JM, Park JM, et al. Clinical features of infectious endophthalmitis in South Korea: a five-year multicenter study. BMC infect Dis 2015;15:177-183.

13. Iylin A, Zhelonkina N, Severskaya N and Romanko S. Nonsurgical Management of thyroid abscess with sonographically guided fine needle aspiration. J Clin Ultrasound 2007;35:333-337.

14. Berger SA, Zonszein J, Villamena P and Mittman N. Infectious diseases of the thyroid gland. Rev Infect Dis 1983;5:108-122.

15. Soni RK, Sinha A. Tuberculosis of the thyroid-a diagnostic enigma. Indian J Surg 2015;77(Suppl 1):179-181.

16. Deepa R, Banu ST, Jayalakshmi G, Parveen JD. Psoas and thyroid abscess in a renal allograft recipient. Indian J Pathol Microbiol 2012;55:593-594.

17. Su BA, Ko WC, Chuang YC, Tang HJ. Disseminated nocardiosis with thyroid involvement: a case report. J Microbiol Immunol Infect 2011;44:238-240.

18. Wang J, Yan Y, Xue X, Wang K, Shen D. Comparison of pyogenic liver abscesses caused by hypermucoviscous Klebsiella pneumoniae and non-Klebsiella pneumoniae pathogens in Beijing: a retrospective analysis. J Int Med Res 2013;41:1088-1097. 